\title{
INFRAESTRUCTURA SOCIAL: MEDICIÓN Y EFECTOS EN EL CRECIMIENTO ${ }^{1}$
}

\section{SOCIAL INFRASTRUCTURE: MEASSURING AND EFFECTS ON GROWTH}

Pablo Francisco Quezada Garza ITESM, Campus Monterrey México pabloqe@gmail.com
José Antonio Marcos Zetter

ITESM, campus Monterrey México marzett15@gmail.com

\section{RESUMEN}

Con base en el artículo de Hall y Jones (1999) el presente estudio comprueba el análisis realizado por estos autores para medir el impacto de la infraestructura social en el producto por trabajador, a través de un índice de infraestructura social distinto y variables instrumentales diferentes. Las principales aportaciones de esta investigación son: (1) el uso de un índice de infraestructura social construido con la técnica de componentes principales y (2) el uso de la tasa de mortalidad de los colonos europeos como variable instrumental. Se encuentra que el efecto de la infraestructura social en el producto por trabajador no es tan grande comparado con lo hallado por Hall y Jones en 1999 (ellos encuentran que un aumento de 1\% en la infraestructura social provoca un incremento de $5.14 \%$ en el producto por trabajador, mientras que en este estudio se estima que el incremento es de $2.46 \%$ o $2.71 \%$, de acuerdo con el índice que se utilice para calcular la regresión). Lo que demuestra este análisis es que, con el mismo marco conceptual de estos autores y con una muestra distinta (Hall y Jones consideran 79 países, mientras que aquí se toman en cuenta 52), un cambio en

1 Los autores agradecen a Edgardo Arturo Ayala Gaytán y Eduardo Enrique Taylor Elizondo por su valiosa contribución en el desarrollo de esta investigación. 
el índice y las variables instrumentales utilizadas provocan una diferencia relativa, en la medida del impacto que tiene la infraestructura social sobre el producto por trabajador.

Palabras clave: infraestructura social, crecimiento económico, variables instrumentales, análisis de componentes principales

Clasificación JEL: O43, C26

\begin{abstract}
Based on Hall \& Jones (1999), this study replicates their analysis which measured the impact of social infrastructure in the income per capita using a different social infrastructure index and different instrumental variables. The use of a social infrastructure index built with the principal components technique plus the use of the mortality rate of the European settlers in America as an instrumental variable, are the main contributions of this research. We find that the impact of social infrastructure on per capita income is not as pronounced as that in the findings of Hall \& Jones in 1999 (Hall and Jones estimate that a 1\% increase in social infrastructure will result in a 5.14\% increase in per capita income while this study estimates a $2.46 \%$ or $2.71 \%$ increase depending on the index used to calculate the regression). What this analysis shows is that, following the same conceptual frame of these authors and using a different sample (Hall and Jones used 79 countries in their study while this analysis considers 52), a change in the index and the instrumental variables used might cause a relative difference in the measure of the impact that social infrastructure has on the income per capita.
\end{abstract}

Keywords: social infrastructure, economic growth, instrumental variables, principal components analysis 


\section{INTRODUCCIÓN}

La infraestructura social (IS) comprende las instituciones y políticas gubernamentales que determinan el ambiente económico en el que los individuos adquieren aptitudes y las empresas se desarrollan (Hall y Jones, 1999). De acuerdo con varios autores, ésta tiene un efecto directo y real en el producto por trabajador (véase Hall y Jones, 1999; Cavalcanti y Novo 2002; Boyle y McQuinn, 2003; Eicher, García y Teksoz, 2006; Glaeser et al., 2004; Rodrik, Subramanian y Trebbi, 2004). Hall y Jones aseguran que este efecto es de hasta $5 \%$ aproximadamente, es decir, que por un cambio porcentual de $1 \%$ en la IS el producto por trabajador aumenta 5 por ciento.

Hall y Jones (1999) identificaron dos problemas para medir el efecto de la IS en el producto por trabajador: (1) no existen medidas conceptualmente válidas en su totalidad para medir la IS y (2) el modelo que comúnmente se utiliza para calcular su impacto en el producto por trabajador presenta un problema de endogeneidad. Para solucionar el primero se pueden construir índices conformados por varios indicadores que, de algún modo, reflejan el nivel de IS de un país. Para solucionar el segundo se puede utilizar la técnica de mínimos cuadrados ordinarios en dos etapas con variables instrumentales. El reto de lo primero está en encontrar indicadores apropiados y con ellos construir un índice apto. El reto de lo segundo está en utilizar las variables instrumentales adecuadas.

Ahora, con base en el marco conceptual de Hall y Jones (1999), ¿qué pasaría si se replica su análisis con índices y variables instrumentales distintas?

Contestar esta pregunta es la motivación del presente estudio. El objetivo es estimar el efecto de la IS sobre el producto por trabajador al emplear herramientas distintas sin salir del marco conceptual propuesto por Hall y 
Jones (1999). Este estudio cuenta con las siguientes aportaciones: el uso de índices conformados por indicadores distintos ponderados con la técnica de componentes principales y variables instrumentales más adecuadas. ${ }^{1}$

Finalmente, se llega a la conclusión de que una variación en la construcción del índice de IS y las variables instrumentales utilizadas para el análisis econométrico puede hacer una diferencia relativa en los resultados obtenidos.

A continuación se presenta el modelo usado para realizar este análisis. Posteriormente se detalla la metodología que se utilizó para construir los índices de infraestructura social. En la sección de selección de instrumentos se mencionan las variables instrumentales que se consideraron para llevar a cabo el estudio. Para concluir, en el apartado de resultados se presentan los efectos que la infraestructura social puede llegar a tener sobre el producto por trabajador de acuerdo con el método de análisis utilizado.

\section{MODELO}

Hall y Jones (1999) aseveran que la IS afecta directamente el producto por trabajador. Para corregir el problema de endogeneidad recurren al método de regresión de mínimos cuadrados ordinarios en dos etapas con variables instrumentales. Como variables instrumentales usan características geográficas como la distancia de un país respecto al ecuador y la fragmentación etnolingüística.

1 De acuerdo con Acemoglu, Robinson y Johnson (2000), las variables instrumentales que se emplean en este estudio son más apropiadas que aquellas utilizadas por Hall y Jones (1999). 
A partir de Hall y Jones (1999) se han escrito varios artículos relacionados y todos ellos justifican la misma relación causal entre la IS y el producto por trabajador: Cavalcanti y Novo (2002) llevan a cabo la misma regresión que Hall y Jones (utilizan los mismos datos y variables instrumentales) con métodos de regresión cuantil y encuentran que los países más pobres aumentan más su producto por trabajador al incrementar $1 \%$ su nivel institucional comparado con los países ricos. Boyle y McQuinn (2003) hacen una prueba de robustez distinta. Hallan que la diferencia del producto por trabajador entre los países se debe, principalmente, a la productividad de los mismos y la IS (medida de acuerdo con Hall y Jones) es significativa y explica la diferencia de productividad de los países. Eicher, García y Teksoz (2006) tratan de determinar si las instituciones afectan, directa o indirectamente, el producto por trabajador. Observan que, indirectamente, lo hacen a través del capital físico y humano. Concluyen que las instituciones son complemento del capital físico y sustituto del humano. Un aumento en el nivel institucional de países con poco capital humano impacta fuertemente el producto por trabajador, a diferencia de los países con mucho capital humano. Finalmente, Glaeser et al. (2004) analizan si las instituciones, en efecto, tienen una relación causal directa con el crecimiento. Entre sus resultados encuentran que, efectivamente, existe una relación entre crecimiento e instituciones; pero es mayor la relación del crecimiento con el capital. Todas estas investigaciones apuntan a que la IS tiene un efecto real en el producto por trabajador.

Por tanto, para medir qué tanto afecta la IS al producto por trabajador se usa el modelo estadístico de Hall y Jones (1999) que se describe brevemente a continuación:

$$
\begin{gathered}
\text { (1) } \log Y / L=\alpha+\beta S+\varepsilon \\
\text { (2) } S=\gamma+\delta \log Y / L+X \theta+\eta
\end{gathered}
$$


Donde $\log Y / L$ se refiere al logaritmo natural del producto por trabajador y $S$ denota la IS. Dado que la IS es determinada endógenamente y depende del nivel de productividad por trabajador se utiliza la matriz $X$ que contiene las variables instrumentales.

La ecuación (1) refleja que la IS es el determinante fundamental y principal de la productividad del trabajador, mientras que la (2) contiene la matriz de variables instrumentales. Cabe recordar que un instrumento adecuado es aquel que afecta directamente a la IS y no afecta el producto por trabajador. Es importante señalar que no es posible medir u observar la IS de manera directa, razón por la cual, igual que Hall y Jones, se recurre a realizar una aproximación mediante variables proxy.

\section{CONSTRUCCIÓN DEL ÍNDICE DE IS}

Medir la IS es un asunto muy complejo y lo presentado en este estudio tiene serios problemas conceptuales. Glaeser, et al. (2004) incluso llegan a descartar este tipo de análisis, porque consideran que el problema de endogeneidad es muy notorio y los instrumentos hasta ahora empleados no son los apropiados. Aun así, en la presente investigación se propone una medida de IS alternativa, que parte de aquella usada por Hall y Jones (1999), y se plantea otro grupo de instrumentos que, de acuerdo con Acemoglu, Robinson y Johnson (2001), son más adecuados.

Para observar la aportación de este artículo, en el cuadro 1 se presentan las diferencias en la construcción del índice de IS del presente estudio y el llevado a cabo por Hall y Jones (1999). Se puede advertir que para cada dimensión descrita por estos últimos se buscó uno o más de un indicador equivalente. Los datos son del Banco Mundial (BM), el Índice de 
Libertad Económica (ILE) y del artículo de Gwartney, Skipton y Lawson (2000). Cabe mencionar que Hall y Jones utilizaron una muestra de 79 países para construir estos índices mientras que aquí se consideran 52 . La muestra usada en este artículo es menor debido a la escasez de información de la tasa de mortalidad de colonos europeos.

\section{Cuadro 1}

Diferencias en la construcción del índice de IS del presente estudio y el realizado por Hall y Jones (1999)

$\begin{aligned} & \text { Indicadores utilizados por Hall y Jones } \\ & \text { (1999) }\end{aligned}$
$\begin{aligned} & \text { Indicadores utilizados en el presente } \\ & \text { artiolo }\end{aligned}$

Ley y orden de International Country $\quad$ Rule of Law, BM

Risk Guide (ICRG)

\begin{tabular}{ll}
\hline Calidad de la burocracia, ICRG & Calidad regulatoria, BM \\
\hline & Efectividad gubernamental, BM \\
\hline Corrupción, ICRG & Control de corrupción, BM \\
\hline Riesgo de expropiación, ICRG & Índice de Libertad de Corrupción, ILE \\
\hline $\begin{array}{l}\text { Incumplimiento de contratos por parte del } \\
\text { gobierno, ICRG }\end{array}$ & Índice de derechos de propiedad, ILE \\
\hline $\begin{array}{l}\text { Índice de Sachs y Warner (1995) para la } \\
\text { apertura económica }\end{array}$ & $\begin{array}{l}\text { Índice de Gwartney, Skipton y Lawson } \\
\text { (2000) para la apertura económica }\end{array}$ \\
\hline & Voice and Accountability, BM \\
\hline
\end{tabular}

Fuente: Elaboración propia. 
Los indicadores del Banco Mundial son resultado de su media aritmética para el rango de años de 1996 a 2010. Esta base de datos se puede consultar en la página web que hace referencia la cita bibliográfica denominada The Worldwide Governance Indicators. Los índices de ILE resultan de su media aritmética para el periodo de 1995 a 2011. Esta base de datos se puede consultar en la página web que hace referencia la cita bibliográfica denominada Index of Economic Freedom. Para construir estos índices se tomaron en cuenta 213 países.

A continuación se presenta un cuadro donde se define cada uno de los indicadores utilizados en este artículo:

\section{Cuadro 2}

Definiciones de los indicadores usados en el presente estudio

\begin{tabular}{ll}
\hline Indicador & Definición \\
\hline & Refleja el grado de confianza que la \\
sociedad le tiene a sus leyes. Se centra \\
en qué tanto se respetan los contratos \\
Rule of Law \\
las cortes.
\end{tabular}

Se refiere a la capacidad que tiene el

Calidad regulatoria gobierno de intrumentar políticas adecuadas para el desarrollo del sector privado.

Muestra la percepción que se tiene de

Efectividad gubernamental los servicios públicos y la calidad de las políticas gubernamentales.

Control de corrupción

Indica qué tanto se utiliza el poder gubernamental para privilegiar algunos intereses privados y cometer crímenes. 
Igual que el indicador de control de corrupción, este índice refleja el abuso

Índice de Libertad de Corrupción, ILE de poder. Se deriva principalmente del Corruption Perceptions Index elaborado por la organización no gubernamental Transparency Internacional.

Se refiere a qué tan probable es que el

Estabilidad política y ausencia del terrorismo gobierno sea reemplazado por medios no constitucionales.

Mide qué tanto se respetan los dere-

Índice de Derechos de Propiedad, ILE chos de propiedad. Incluye también la probabilidad de que el gobierno pueda expropiarla.

Fuente: elaboración propia.

Se puede notar que se agregó la dimensión de "Voice and Accountability”, que se refiere a la capacidad que tienen los ciudadanos para ser escuchados y pedir la rendición de cuentas a sus gobernantes. Se esperaría que una buena IS les permitiera a los ciudadanos ser escuchados y hacer que sus gobernantes rindan cuentas. Se incluye esta dimensión porque se cree que la naturaleza de la IS es muy amplia y multidimensional, por tanto, entre más información haya para sus dimensiones el índice de IS será de mejor calidad.

En cuanto al indicador de apertura económica se utiliza el de Gwartney, Skipton y Lawson (2000) en lugar de aquel sugerido por Hall y Jones (1999), debido a que se consideró que el utilizado por éstos es menos objetivo que el de ellos. Hall y Jones (1999) usan el índice construido por Sachs y Warner (1995), que mide el lapso entre 1950 y 1994 cuando el país estuvo abierto; se considera que un país está abierto si cumple con los siguientes requisitos: (1) sus barreras no arancelarias cubren menos de $40 \%$ del comercio internacional, (2) la tasa promedio de los aranceles es menor a 40\%, (3) cualquier prima del mercado negro era inferior a $20 \%$ 
durante los años 1970 y 1980, (4) el país no es considerado socialista por Kornai (1992) y (5) el gobierno no monopoliza las principales exportaciones. En cambio, el índice de Gwartney, Skipton y Lawson (2000) es un poco más sencillo y, en nuestra opinión, más objetivo, ya que no tiene criterios arbitrarios, como establecer que un país no estuvo abierto un año por tener barreras no arancelarias que cubrían más de 40\% del comercio internacional. Este índice tiene cuatro dimensiones: (1) tasas arancelarias, (2) la prima del mercado negro de divisas, (3) restricciones en los movimientos de capital y (4) el tamaño del comercio internacional comparado con el tamaño esperado de acuerdo con las características de cada país ${ }^{2}$. El índice está construido con el promedio de los datos del periodo que abarca de 1980 a 1997, para 97 países.

Con la técnica de componentes principales se construyeron dos índices de IS: el índice A y el índice B. Al tomar en cuenta que ambos se calculan con los productos de la misma técnica, se espera que los resultados de la regresión, al utilizar el índice $\mathrm{A}$, sean parecidos a los resultados de la regresión, al utilizar el índice B. Con esto se busca probar que una variación en el criterio para utilizar los resultados del análisis multivariado no altera considerablemente el efecto de la IS en el producto por trabajador.

El índice A se calculó de la siguiente manera:

$\sum_{i=1}^{n}($ carga del factor de la matriz no rotada) (indicador de la observación)

2 El tamaño esperado del comercio internacional está en función del tamaño del país (medido en población con edad de trabajar), la localización del mismo y el producto interno bruto mundial. Para más detalles véase Gwartney, Skipton y Lawson (2000). 
El índice B se calculó de la siguiente manera:

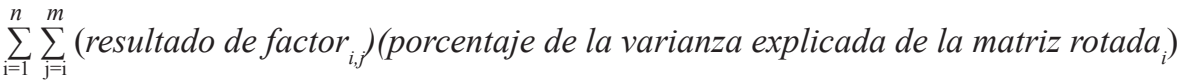

La varianza total explicada, la matriz de componentes no rotada y el resultado de factores que se utilizaron para calcular estos índices se encuentran en la sección de anexos.

Una ventaja del método usado en el presente estudio es que toma en cuenta el peso que tiene cada variable respecto a las demás. Así se evita asumir que cada variable tiene la misma ponderación en la composición de la IS. Los índices tienen un rango de cero a uno, donde un número cercano a uno significa que el país cuenta con una buena IS, y un número cercano a cero, que el país cuenta con una mala IS.

\section{SELECCIÓN DE INSTRUMENTOS}

Como se verá a continuación, en el presente estudio se utilizan dos variables instrumentales: distancia del ecuador y tasa de mortalidad de los colonos europeos. Resulta conveniente usar dichas variables como instrumentos ya que cuentan con el respaldo de diversos autores. Por ejemplo, Acemoglu, Johnson y Robinson (2001) mencionan que la influencia europea en sus colonias fue una fuente de crecimiento. Donde los europeos encontraban un lugar en condiciones para habitar-generalmente buscaban ecosistemas similares a los de sus países de origen-imponían su sistema institucional. En cambio, en los lugares donde no encontraban ecosistemas propicios establecían un sistema institucional extractivo y, por ende, inadecuado para el desarrollo de la región. Por tal razón, estos autores 
proponen manejar la tasa de mortalidad de los colonos europeos como instrumento para medir el impacto de la IS en el producto por trabajador. Por otro lado, Easterly y Levine (2003) encuentran que la localización, las condiciones ecológicas y la posibilidad de plantar ciertas cosechas sólo afectan el desarrollo económico a través de las instituciones. Lo que indica que la distancia del ecuador es una buena variable instrumental, ya que está correlacionada con la IS y no con el producto por trabajador. Finalmente, cabe recalcar que Sachs (2003) recurre a la distancia del ecuador como variable instrumental de la IS.

En el cuadro 2 se comparan los instrumentos utilizados por Hall y Jones (1999) y los del presente artículo.

\section{Cuadro 3}

Variables instrumentales utilizadas por Hall y Jones (1999) y las consideradas en el presente articulo

\begin{tabular}{l|l}
\hline $\begin{array}{l}\text { Variables instrumentales usadas por Hall y } \\
\text { Jones (1999) }\end{array}$ & $\begin{array}{l}\text { Variables instrumentales usadas en el } \\
\text { presente artículo }\end{array}$ \\
\hline $\begin{array}{l}\text { Distancia del ecuador } \\
\text { Distancia del ecuador (OpenData: } \\
\text { Country list ISO 3166 codes latitude } \\
\text { longitude) }\end{array}$ \\
\hline $\begin{array}{l}\text { Idioma } \\
\begin{array}{l}\text { Proporción del comercio de una economía } \\
\text { llado por Frankel y Romer (1996). }\end{array}\end{array}$ & $\begin{array}{l}\text { Tasa de mortalidad de los colonos euro- } \\
\text { peos (Acemoglu, Johnson y Robinson, } \\
2001) .\end{array}$ \\
\hline
\end{tabular}

Fuente: Elaboración propia.

Se excluye el instrumento del idioma porque resulta inapropiado debido a que tiene efectos causales directos con la variable dependiente. Se mantiene la distancia del ecuador y se agrega la tasa de mortalidad de los colonos europeos. 


\section{RESULTADOS}

En el siguiente cuadro se presentan los resultados de esta investigación

\section{Cuadro 4}

Resultados

$\log (\mathrm{Y} / \mathrm{L})=\alpha+\beta \tilde{S^{2}}+\tilde{\varepsilon}$

\section{Método de estimación}

\section{Mínimos cuadrados ordinarios}

Mínimos cuadrados ordinarios en dos etapas con variables instrumentales

\begin{tabular}{lcc}
\hline Modelo A & & \\
Constante & $2.585096 * * *$ & $2.319469 * * *$ \\
& $(-0.099598)$ & $(-0.159727)$ \\
Índice de IS A & $2.104029 * * *$ & $2.711334 * * *$ \\
& $(-0.19911)$ & $(-0.344793)$ \\
Número de observaciones & 52 & 52 \\
R cuadrada & 0.690718 & 0.633172 \\
\hline Modelo B & & $2.540626 * * *$ \\
Constante & $2.838372 * * *$ & $(-0.161913)$ \\
& $(-0.099075)$ & $2.469429 * * *$ \\
Índice de IS B & $1.707293 * * *$ & $(-0.380834)$ \\
& $(-0.207951)$ & 52 \\
Número de observaciones & 52 & 0.459718 \\
R cuadrada & 0.574125 &
\end{tabular}

Notas: Errores estándar en paréntesis; *** significancia a 1 por ciento. 
El modelo A se refiere al que utilizó el índice A y el modelo B se refiere al que empleó el índice $\mathrm{B}$.

Como se puede observar en el cuadro 4, se calculó el modelo estructural con dos métodos de estimación y dos índices de IS distintos. Se incluyeron las estimaciones con el método de mínimos cuadrados ordinarios para comparar los resultados obtenidos por el método de mínimos cuadrados en dos etapas con variables instrumentales y tener una referencia. Tal como lo indican los asteriscos, todas las estimaciones son significativas.

La interpretación de los resultados es la siguiente: al usar el índice A y los instrumentos, por cada $1 \%$ que aumente la IS el producto por trabajador aumentará 2.71\%; y al considerar el índice B y los instrumentos, por cada $1 \%$ que aumente la IS el producto por trabajador aumentará 2.46 por ciento.

Hall y Jones (1999) encuentran que un incremento de $1 \%$ en la IS resulta un aumento de $5.14 \%$ en el producto por trabajador. Por lo tanto, de acuerdo con los resultados de este estudio, se puede decir que, con el mismo marco conceptual de dichos autores, un cambio en el índice y las variables instrumentales pueden hacer una diferencia relativa en la medida del impacto que tiene la IS en el producto por trabajador.

\section{CONCLUSIONES}

La IS es un concepto difícil de medir. Autores como Hall y Jones (1999) han intentado medir su incidencia en el crecimiento económico y han sido fuertemente criticados. En el presente artículo se intenta mejorar la medición del efecto que tiene este concepto en el producto por trabajador, al utilizar un índice de IS distinto y variables instrumentales que, de acuerdo con Acemoglu, Robinson y Johnson (2001), son apropiadas para dicho fin. 
Se encontró que, a diferencia de lo observado por Hall y Jones (1999), el impacto de la IS en el crecimiento económico no es tan grande. En este estudio se encuentra que el impacto de la IS en el producto por trabajador es de $2.71 \%$ al utilizar el índice A y $2.46 \%$ con el índice B, mientras que Hall y Jones (1999) hallan un efecto de 5.14 por ciento.

Cabe recalcar que el uso de mejores instrumentos y un índice de IS distinto no contradice la hipótesis original de la IS, pero sí demuestra que, dentro del mismo marco conceptual, un cambio en el índice y las variables instrumentales utilizadas pueden resultar medidas relativamente distintas del impacto de la IS sobre el producto por trabajador.

Una limitante de este estudio es la cantidad de países que se analizaron. A diferencia de Hall y Jones que utilizan 79 países para realizar su investigación, se consideraron solamente 52, debido a la escasez de datos de la tasa de mortalidad de los colonos europeos. La diferencia de los resultados obtenidos puede deberse a esta condición. En futuras investigaciones habrá que usar una variable instrumental que permita incluir más países al estudio.

La principal limitante de nuestro análisis radica en la dificultad de medir la IS y el problema de endogeneidad que se genera a la hora de ver su efecto en el producto por trabajador. El modelo presentado tiene serios problemas conceptuales que se tratan de atenuar con el uso de las mejores variables instrumentales disponibles. Para futuras investigaciones queda seguir mejorando la medida de la IS y sus instrumentos, en caso de que sean necesarios para estimar su impacto en el producto por trabajador. 


\section{REFERENCIAS}

Acemoglu, D., S. Johnson.y J.A. Robinson. 2001. "The colonial origins of comparative development: An empirical investigation", The American Economic Review, 91(5): 1369-1401.

Banco Mundial. s/f. The Worldwide Governance Indicators, <http://info. worldbank.org/governance/wgi/sc_country.asp $>$.

Boyle, G. y K. McQuinn. 2003. "Why do some countries produce so much more output per worker than others? - A note", The Quarterly Journal of Economics, 114 (1): 83-116.

Cavalcanti, T.V.D.V. y A.A. Novo. 2002. "Institutions and economic development: how strong is the relation?", Banco de Portugal, DT 200213.

Easterly, W. y R. Levine. 2003. "Tropics, germs, and crops: how endowments influence economic development", Journal of Monetary Economics, 50: 3-39.

Eicher, T., C. Garcia-Peñalosa and U. Teksoz. 2006. "How do institutions lead some countries to produce so much more output per worker than others?" en T. Eicher y C. Garcia-Peñalosa: Institutions, development, and economic growth, MIT Press, Cambridge, pp. 65-80.

Frankel, Jeffrey A. y David Romer, "Trade and Growth: An Empirical Investigation", Marzo 1996. NBER Working Paper No. 5476

Glaeser, E., R. La Porta, F. López-de-Silanes y A. Shleifer. 2004. "Do institutions cause growth?”, NBER, Working Paper Series, núm. 10568, Cambridge.

Gwartney, J., C. Skipton. y R. Lawson. 2000. “Trade, openness and economic growth", manuscrito presentado en Southern Economics Association Annual Meetings, nov. 2000. 
Hall, R. y C. Jones. 1999. "Why do some countries produce so much more output than others?", The Quarterly Journal of Economics, 114(1): 83-116.

Heritage Foundation. s/f. Index of economic freedom, $<$ http://www.heritage.org/index/>.

Kaufmann, D., A. Kraay y M. Mastruzzi. 2010. Worldwide governance indicators (WGI) project, Banco Mundial, <http://info.worldbank. org/governance/wgi/pdf/wgidataset.xls $>$.

Kornai, J. 1992. The socialist system: The political economy of communism, Princeton University Press.

Socrata. s/f. OpenData: Country list ISO 3166 codes latitude longitude, $<$ https://opendata.socrata.com/dataset/Country-List-ISO-3166-CodesLatitude-Longitude/mnkm-8ram>.

Rodrik, D., A. Subramanian y F. Trebbi. 2004. "Institutions rule: the primacy of institutions over geography and integration in economic development”, NBER, Working Paper Series, núm. 9305, Cambridge.

Sachs, J. 2003. "Institutions don't rule: direct effects of geography on per capita income", NBER, Working Paper Series, núm. 9490, Cambridge.

. y A. Warner. 1995. "Economic Reform and the Process of Global Integration", Brookings Papers on Economic Activity 1:1995, Washington. 


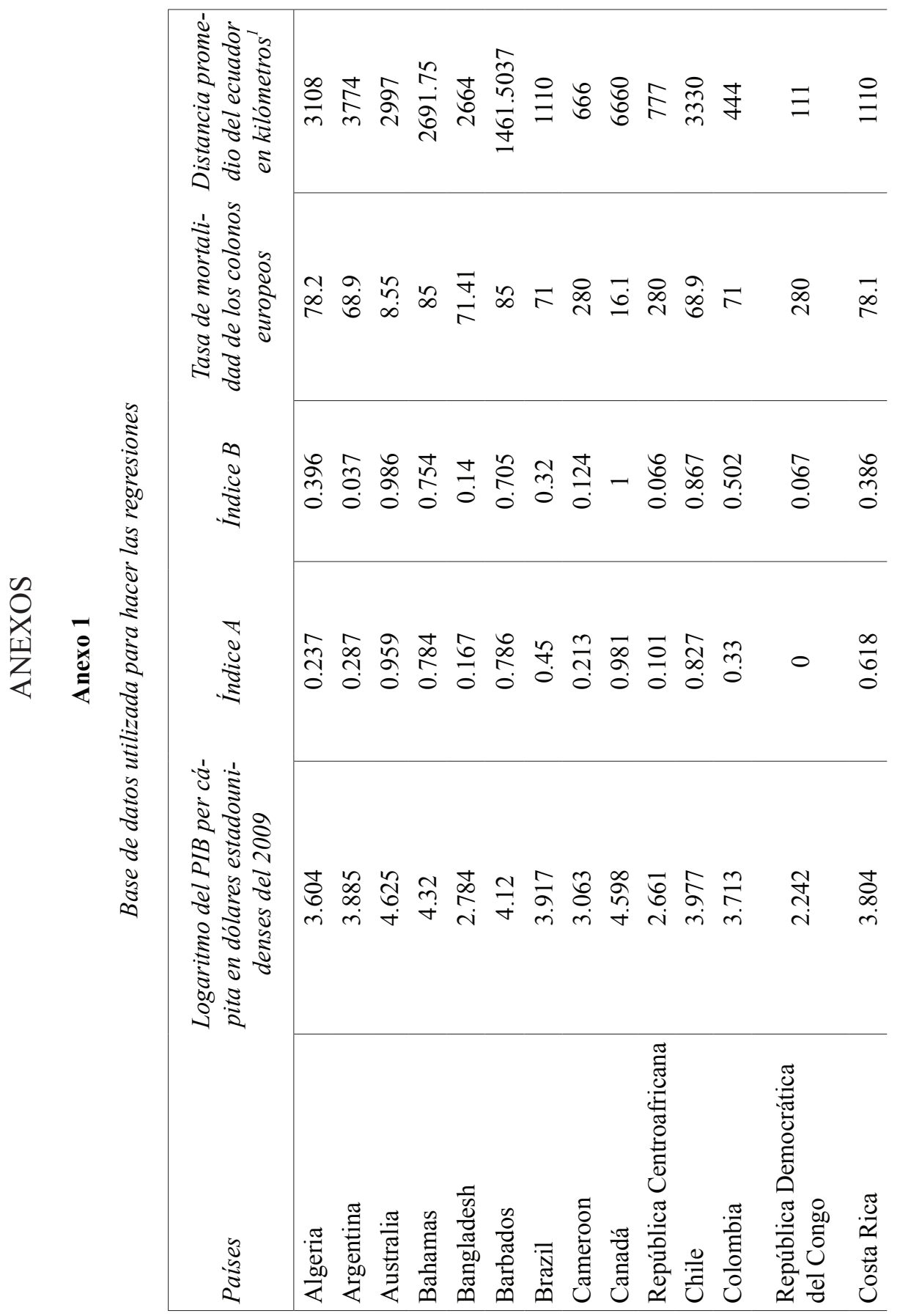

ISSN 0188-266X - Revista de Economía - Vol. XXXI - Núm. 82 


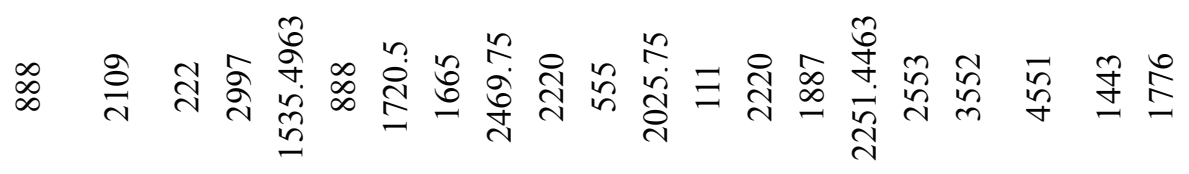

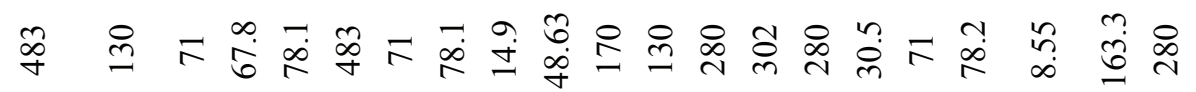

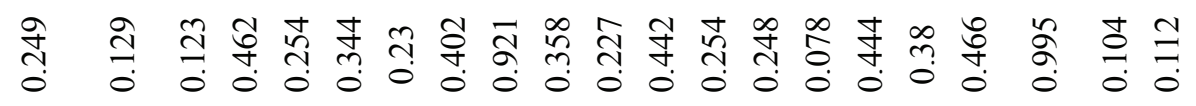

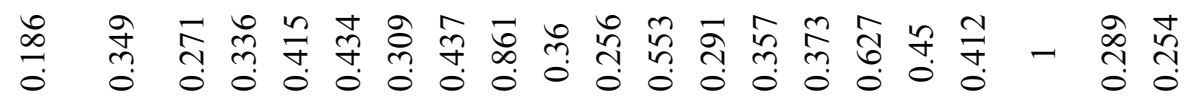

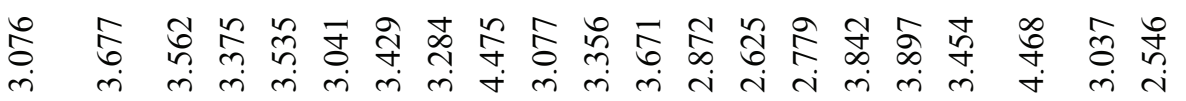

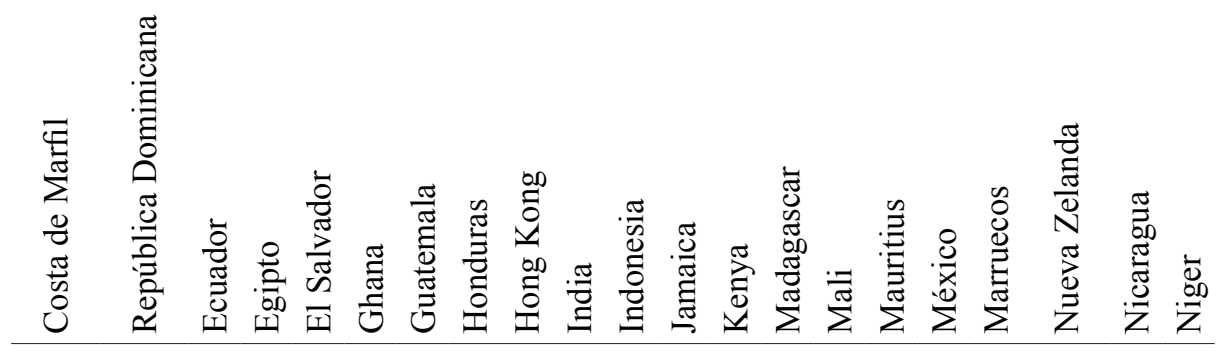

Enero - junio de 2014 - Págs. 11-36 


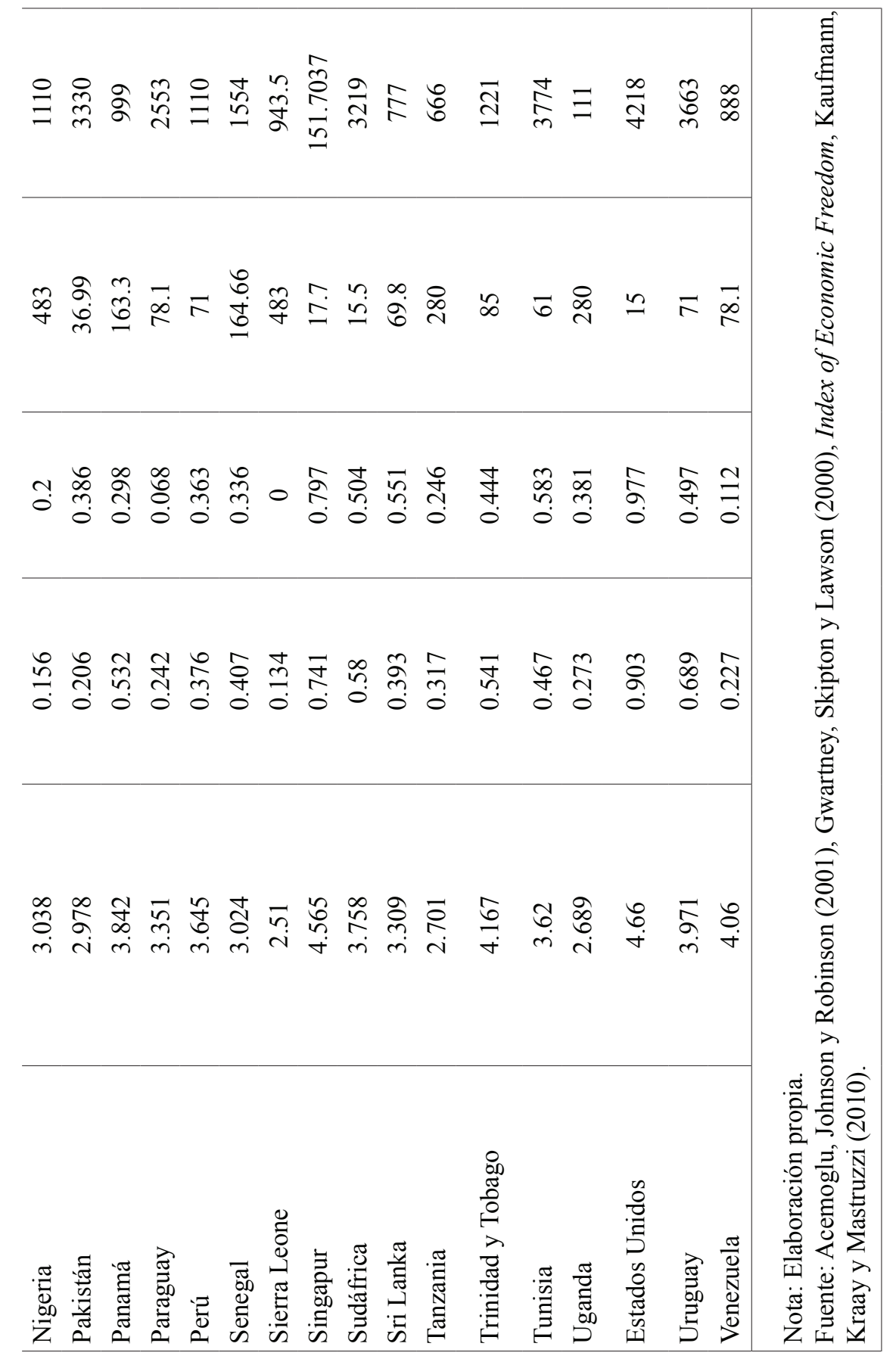

ISSN 0188-266X - Revista de Economía - Vol. XXXI - Núm. 82 


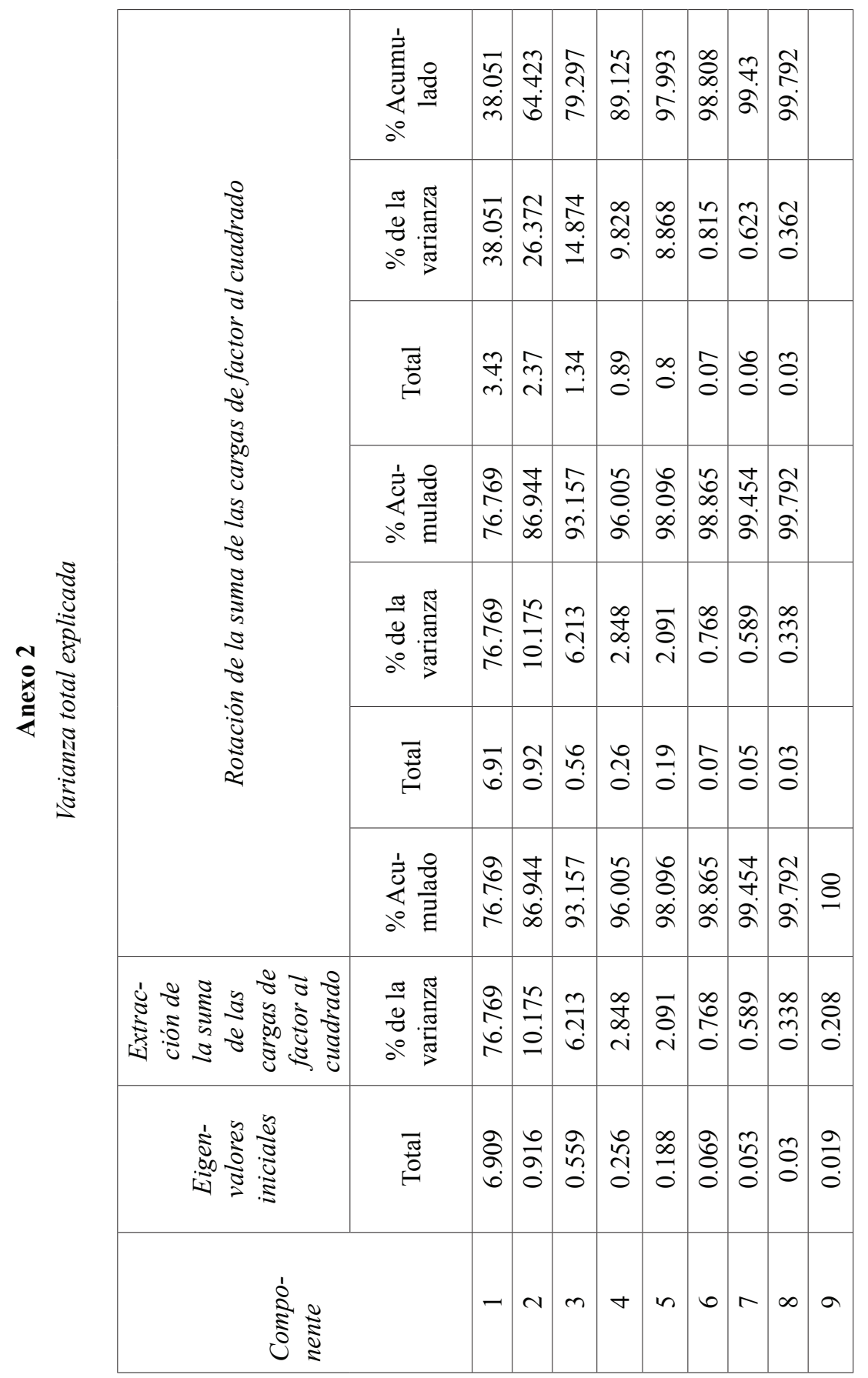

Enero - junio de 2014 - Págs. 11-36 


\section{Anexo 3}

\begin{tabular}{|c|c|c|c|c|c|c|c|c|}
\hline \multicolumn{9}{|c|}{ Matriz de componentes no rotada } \\
\hline & \multicolumn{8}{|c|}{ Componentes } \\
\hline Variables & 1 & 2 & 3 & 4 & 5 & 6 & 7 & 8 \\
\hline $\begin{array}{l}\text { Voice and } \\
\text { Accounta- } \\
\text { bility }\end{array}$ & 0.885 & 0.111 & -0.177 & 0.307 & -0.277 & 0.051 & 0.005 & -0.003 \\
\hline $\begin{array}{l}\text { Estabilidad } \\
\text { política y } \\
\text { ausencia } \\
\text { del terroris- } \\
\text { mo }\end{array}$ & 0.868 & -0.099 & -0.305 & 0.23 & 0.298 & -0.041 & -0.019 & 0.011 \\
\hline $\begin{array}{l}\text { Efectividad } \\
\text { guberna- } \\
\text { mental }\end{array}$ & 0.957 & -0.168 & -0.063 & -0.175 & -0.052 & -0.016 & -0.07 & 0.092 \\
\hline $\begin{array}{l}\text { Calidad } \\
\text { regulatoria }\end{array}$ & 0.961 & -0.136 & 0.024 & -0.076 & -0.107 & -0.179 & -0.052 & -0.026 \\
\hline $\begin{array}{l}\text { Rule of } \\
\text { Law }\end{array}$ & 0.952 & -0.127 & -0.146 & -0.157 & 0.004 & 0.088 & 0.127 & 0.054 \\
\hline $\begin{array}{l}\text { Control de } \\
\text { corrupción }\end{array}$ & 0.956 & -0.177 & -0.091 & -0.15 & 0.022 & 0.073 & -0.006 & -0.134 \\
\hline $\begin{array}{l}\text { Índice de } \\
\text { Gwartney, } \\
\text { Skipton y } \\
\text { Lawson } \\
\text { (2000) para } \\
\text { la apertura } \\
\text { económica }\end{array}$ & 0.664 & -0.451 & 0.572 & 0.155 & 0.039 & 0.024 & 0.027 & 0.008 \\
\hline $\begin{array}{l}\text { El índice } \\
\text { de dere- } \\
\text { chos de } \\
\text { propiedad }\end{array}$ & 0.785 & 0.574 & 0.17 & -0.019 & 0.039 & -0.093 & 0.118 & -0.01 \\
\hline $\begin{array}{l}\text { Índice de } \\
\text { libertad de } \\
\text { corrupción }\end{array}$ & 0.811 & 0.516 & 0.211 & -0.032 & 0.071 & 0.1 & -0.119 & 0.011 \\
\hline
\end{tabular}

ISSN 0188-266X - Revista de Economía - Vol. XXXI - Núm. 82 


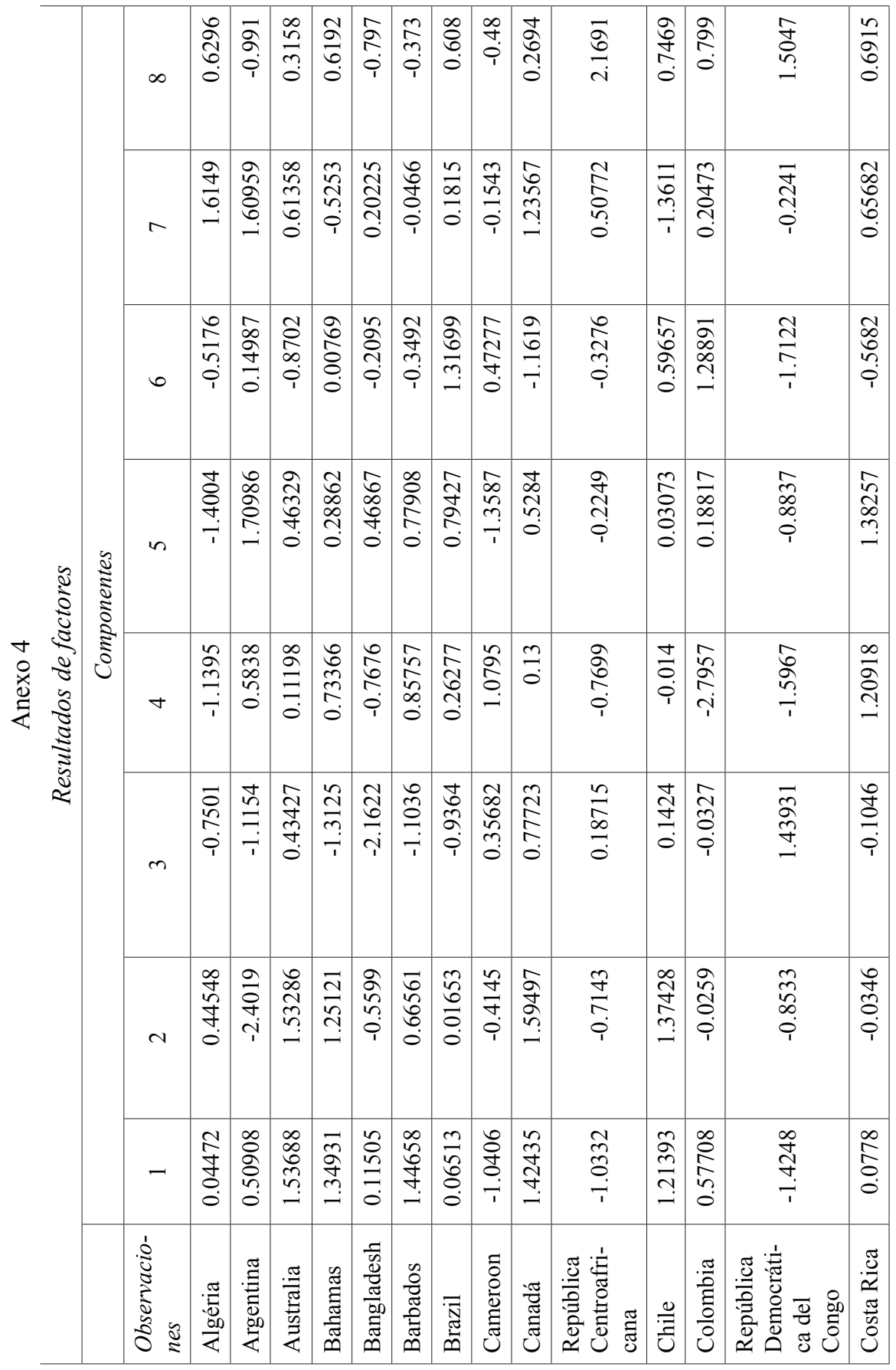

Enero - junio de 2014 - Págs. 11-36 


\begin{tabular}{|c|c|c|c|c|c|c|c|c|c|c|c|c|c|c|c|c|c|c|c|c|}
\hline$\stackrel{n}{n}$ & $\begin{array}{l}\text { O̦ } \\
\stackrel{0}{0}\end{array}$ & $\overrightarrow{p_{1}}$ & $\mid \begin{array}{l}n \\
\tilde{o} \\
i \\
1\end{array}$ & $\mid \begin{array}{c}0 \\
0 \\
0 \\
0 \\
1\end{array}$ & $\begin{array}{l}n \\
0 \\
0 \\
0\end{array}$ & \begin{tabular}{l}
$\mathfrak{n}$ \\
\multirow{J}{0}{} \\
$\dot{0}$
\end{tabular} & $\begin{array}{l}\overline{0} \\
0 \\
0 \\
1\end{array}$ & 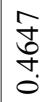 & 午 & ָे & $\begin{array}{l}0 \\
n \\
n \\
\\
1\end{array}$ & م્ & $\begin{array}{l}\hat{\infty} \\
\tilde{0} \\
\dot{1}\end{array}$ & $\begin{array}{l}m \\
\hat{n} \\
\tilde{n}\end{array}$ & $\stackrel{\widehat{ָ}}{\underset{T}{1}}$ & $\frac{m}{a}$ & 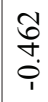 & $\begin{array}{l}0 \\
0 \\
0 \\
\\
1\end{array}$ & $\begin{array}{l}\vec{\infty} \\
\infty \\
\infty \\
-\end{array}$ & $\frac{a}{0}$ \\
\hline$\stackrel{\infty}{\stackrel{\infty}{0}}$ & $\begin{array}{l}\infty \\
\infty \\
n \\
= \\
=\end{array}$ & $\frac{\mathfrak{\sigma}}{\tilde{m}}$ & $\begin{array}{l}\exists \\
\dot{T} \\
\end{array}$ & 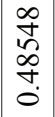 & $\begin{array}{l}\text { J } \\
\text { J } \\
0 \\
0\end{array}$ & 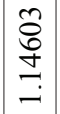 & 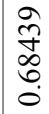 & 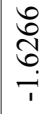 & 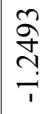 & $\begin{array}{l}n \\
8 \\
\\
\end{array}$ & 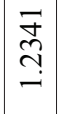 & $\begin{array}{l} \pm \\
\hat{\sigma} \\
\hat{\alpha}\end{array}$ & 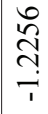 & $\begin{array}{l}n \\
\infty \\
\infty \\
i \\
i\end{array}$ & \begin{tabular}{l}
$\vec{\infty}$ \\
0 \\
$\stackrel{0}{1}$ \\
\hdashline
\end{tabular} & $\mid \begin{array}{l}\infty \\
\stackrel{2}{\widehat{C}} \\
0 \\
\dot{0}\end{array}$ & 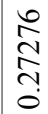 & $\begin{array}{l}\vec{\infty} \\
\stackrel{\infty}{+} \\
\stackrel{-}{\leftrightarrows}\end{array}$ & 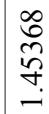 & $\begin{array}{l}\infty \\
n \\
= \\
0\end{array}$ \\
\hline $\begin{array}{l}m \\
\infty \\
2 \\
\tilde{n} \\
0\end{array}$ & $\begin{array}{l}\hat{n} \\
\hat{n} \\
\tilde{n} \\
0\end{array}$ & $\begin{array}{l}\underset{J}{J} \\
\infty \\
\dot{0} \\
1\end{array}$ & $\left|\begin{array}{l}m \\
\infty \\
n \\
\dot{p} \\
1\end{array}\right|$ & $\begin{array}{l}n \\
\widetilde{Z} \\
\widehat{Z} \\
\end{array}$ & $\frac{\mathfrak{0}}{\stackrel{n}{n}}$ & 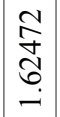 & 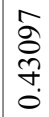 & 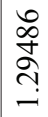 & 光 & $\begin{array}{l}2 \\
\infty \\
0 \\
0 \\
0 \\
1\end{array}$ & $\begin{array}{l}\hat{2} \\
2 \\
2 \\
0 \\
0\end{array}$ & $\begin{array}{l}\infty \\
\tilde{\infty} \\
\infty \\
\hat{\alpha} \\
0\end{array}$ & $\begin{array}{l}n \\
\tilde{o} \\
0 \\
0\end{array}$ & 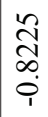 & 言 & $\begin{array}{l}\bar{\delta} \\
\dot{\delta} \\
\dot{-}\end{array}$ & $\begin{array}{l}\vec{\infty} \\
0 \\
0 \\
0 \\
1\end{array}$ & 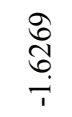 & $\begin{array}{l}\sigma \\
\infty \\
0 \\
\dot{0}\end{array}$ & 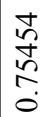 \\
\hline 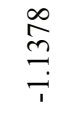 & $\begin{array}{l}\vec{\sigma} \\
\stackrel{0}{n} \\
\stackrel{0}{0}\end{array}$ & $\begin{array}{l}0 \\
\infty \\
\infty \\
+ \\
0\end{array}$ & 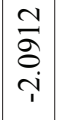 & \begin{tabular}{|l|}
$\infty$ \\
$\widetilde{6}$ \\
0 \\
0 \\
0
\end{tabular} & $\begin{array}{l}\overrightarrow{0} \\
\text { ț } \\
\\
0\end{array}$ & 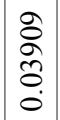 & $\begin{array}{l}\vec{m} \\
\stackrel{m}{\longrightarrow}\end{array}$ & $\frac{1}{\stackrel{1}{+}}$ & $\begin{array}{l}\stackrel{n}{n} \\
\stackrel{n}{2}\end{array}$ & $\begin{array}{l}+ \\
\infty \\
\delta \\
\infty \\
\infty \\
0\end{array}$ & 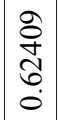 & 光 & 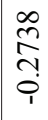 & $\begin{array}{l}\infty \\
\infty \\
\infty \\
\infty \\
0\end{array}$ & $\begin{array}{l}\hat{n} \\
\text { ơ } \\
\text { ó }\end{array}$ & $\begin{array}{l}\mathfrak{n} \\
\hat{2} \\
\hat{2} \\
0\end{array}$ & $\frac{\mathcal{F}}{\mathcal{J}}$ & $\begin{array}{l}\stackrel{0}{*} \\
\stackrel{+}{n} \\
\end{array}$ & 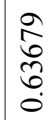 & $\begin{array}{l}\infty \\
\text { مे } \\
\text { กे } \\
0\end{array}$ \\
\hline $\begin{array}{l}\tilde{n} \\
\hat{\Omega} \\
\stackrel{0}{0}\end{array}$ & 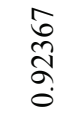 & 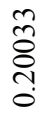 & $\mid \begin{array}{c}\infty \\
\stackrel{N}{\mathfrak{N}} \\
\stackrel{1}{0}\end{array}$ & $\begin{array}{c}\infty \\
\stackrel{\infty}{\delta} \\
\stackrel{\delta}{\delta} \\
\end{array}$ & $\begin{array}{l}0 \\
\bar{n} \\
n \\
0 \\
0\end{array}$ & $\frac{n}{n}$ & $\begin{array}{l}\frac{1}{\delta} \\
0 \\
0\end{array}$ & $\begin{array}{l}\text { ते } \\
\text { ర్} \\
0\end{array}$ & 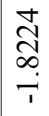 & 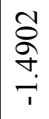 & $\begin{array}{c}0 \\
0 \\
\tilde{N} \\
\infty \\
0 \\
0 \\
0\end{array}$ & 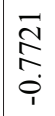 & 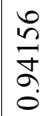 & 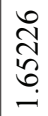 & \begin{tabular}{l}
$\infty$ \\
8 \\
8 \\
\hdashline \\
\end{tabular} & $\begin{array}{l}n \\
\tilde{2} \\
\tilde{n} \\
0 \\
1\end{array}$ & $\begin{array}{l}0 \\
\infty \\
0 \\
\infty \\
1 \\
0\end{array}$ & $\begin{array}{l}\overrightarrow{0} \\
\infty \\
0 \\
0\end{array}$ & 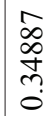 & 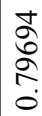 \\
\hline$\frac{\stackrel{0}{a}}{\stackrel{2}{*}}$ & $\frac{\circ}{\stackrel{\circ}{\circ}}$ & $\underset{m}{m}$ & $\mid \begin{array}{l}\infty \\
n \\
n \\
n \\
0 \\
1\end{array}$ & $\begin{array}{l}\infty \\
\infty \\
\tilde{n} \\
\tilde{n} \\
\hat{1}\end{array}$ & 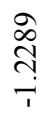 & $\begin{array}{l} \pm \\
\text { in } \\
0 \\
0 \\
0\end{array}$ & $\frac{a}{\sigma}$ & $\begin{array}{l}\text { oे } \\
\text { ळे } \\
\text { ते }\end{array}$ & $\begin{array}{l}n \\
\infty \\
\infty \\
0 \\
0\end{array}$ & $\begin{array}{l}n \\
n \\
0 \\
0 \\
-1\end{array}$ & 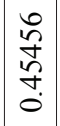 & 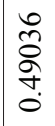 & $\begin{array}{l}\hat{n} \\
\hat{\alpha} \\
\hat{1}\end{array}$ & 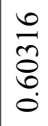 & \begin{tabular}{l}
0 \\
\multirow{2}{N}{} \\
0 \\
1
\end{tabular} & $\begin{array}{c}0 \\
\stackrel{1}{N} \\
m \\
\mathfrak{m} \\
0\end{array}$ & \begin{tabular}{l}
$\infty$ \\
\multirow{2}{n}{} \\
$n$ \\
0
\end{tabular} & $\begin{array}{l}m \\
\stackrel{n}{+} \\
i \\
i\end{array}$ & $\begin{array}{l}\infty \\
\infty \\
\infty \\
\tilde{n} \\
\tilde{0}\end{array}$ & $\begin{array}{l}\infty \\
0 \\
0 \\
0 \\
1 \\
1\end{array}$ \\
\hline $\begin{array}{l}0 \\
0 \\
\infty \\
0 \\
0 \\
1\end{array}$ & $\begin{array}{l}2 \\
\hat{n} \\
n \\
0\end{array}$ & $\begin{array}{l}\vec{N} \\
\tilde{n} \\
\stackrel{n}{o}\end{array}$ & 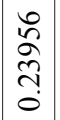 & $\begin{array}{c}\vec{z} \\
\tilde{a} \\
\tilde{n} \\
0\end{array}$ & 品 & $\begin{array}{c}\tilde{\sigma} \\
\stackrel{0}{0}\end{array}$ & $\begin{array}{l}2 \\
\infty \\
\infty \\
\infty \\
i\end{array}$ & $\frac{\partial}{\partial}$ & 官 & 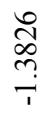 & $\begin{array}{l}\widehat{N} \\
\tilde{N} \\
\infty \\
\infty \\
-\end{array}$ & 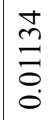 & 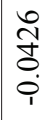 & 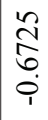 & $\begin{array}{l}\vec{n} \\
\stackrel{n}{0} \\
\stackrel{1}{1}\end{array}$ & $\begin{array}{l}\hat{n} \\
\hat{o} \\
0 \\
0\end{array}$ & $\frac{\infty}{\stackrel{0}{巳}}$ & $\begin{array}{l}\stackrel{n}{\infty} \\
\stackrel{+}{+} \\
\stackrel{1}{1}\end{array}$ & 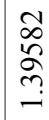 & 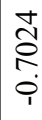 \\
\hline $\begin{array}{l}\exists \\
\text { J } \\
\dot{0} \\
\dot{1}\end{array}$ & $\begin{array}{l}\hat{\infty} \\
\hat{\sigma} \\
\dot{0} \\
1\end{array}$ & $\begin{array}{c}\infty \\
\infty \\
\stackrel{\infty}{+}\end{array}$ & $\left|\begin{array}{c}0 \\
\text { oे } \\
\infty \\
\dot{0} \\
\dot{+} \\
\dot{0}\end{array}\right|$ & $\begin{array}{c}\vec{n} \\
\hat{n} \\
0 \\
\dot{1}\end{array}$ & $\frac{\infty}{0}$ & 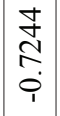 & 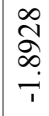 & 吉 & $\frac{2}{2}$ & $\frac{0}{2}$ & $\begin{array}{c}\stackrel{2}{2} \\
\stackrel{1}{-1}\end{array}$ & $\begin{array}{l}+ \\
\frac{1}{9} \\
\dot{0}\end{array}$ & 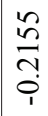 & 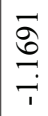 & $\frac{n}{n}$ & $\begin{array}{l}n \\
\tilde{a} \\
\dot{0} \\
1\end{array}$ & 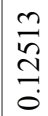 & $\frac{\stackrel{Y}{ \pm}}{\stackrel{+}{+}}$ & $\begin{array}{l}\infty \\
\text { సे } \\
\text { శె} \\
\stackrel{-}{-}\end{array}$ & $\begin{array}{l}\infty \\
\text { N } \\
\infty \\
n \\
0 \\
1\end{array}$ \\
\hline 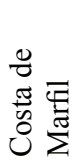 & 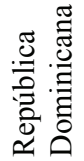 & 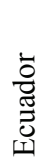 & $\mid \begin{array}{l}\stackrel{0}{0} \\
.00 \\
.01\end{array}$ & 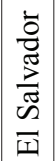 & 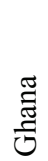 & 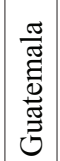 & 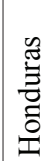 & $\begin{array}{l}00 \\
\tilde{0} \\
1 \\
0 \\
00 \\
0 \\
0 \\
0\end{array}$ & 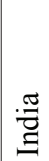 & 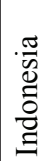 & 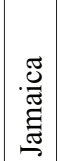 & 节 & 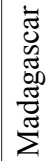 & $\sum_{i}^{i=}$ & 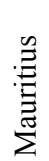 & 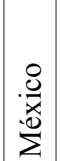 & 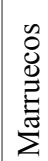 & 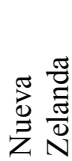 & 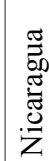 &. \\
\hline
\end{tabular}

ISSN 0188-266X - Revista de Economía - Vol. XXXI - Núm. 82 


\begin{tabular}{|c|c|c|c|c|c|c|c|c|c|c|c|c|c|c|c|c|c|}
\hline $\begin{array}{l}m \\
\stackrel{2}{\Xi} \\
\dot{0}\end{array}$ & $\begin{array}{l}\hat{\sigma} \\
\vec{J} \\
\stackrel{0}{0}\end{array}$ & $\begin{array}{l}0 \\
2 \\
\infty \\
\dot{1} \\
1\end{array}$ & 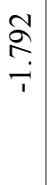 & $\frac{n}{R}$ & 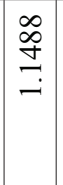 & $\frac{m}{\tilde{N}}$ & $\begin{array}{l}\hat{\sigma} \\
\text { ŏ }\end{array}$ & $\begin{array}{l}\tilde{2} \\
\tilde{2} \\
\tilde{n} \\
0\end{array}$ & \begin{tabular}{|l|} 
\\
$\infty$ \\
1 \\
1 \\
0
\end{tabular} & $\begin{array}{l}\overrightarrow{\bar{N}} \\
\stackrel{1}{1} \\
\stackrel{1}{1}\end{array}$ & $\frac{N}{\sigma}$ & $\begin{array}{l}0 \\
\stackrel{2}{0} \\
0 \\
\end{array}$ & $\begin{array}{l}2 \\
\delta \\
i \\
i\end{array}$ & $\begin{array}{l}\text { तु } \\
\stackrel{0}{0} \\
\dot{1}\end{array}$ & \begin{tabular}{l|}
$\frac{m}{R}$ \\
$\hat{0}$ \\
$\dot{1}$
\end{tabular} & $\begin{array}{l}\infty \\
n \\
n \\
n\end{array}$ & 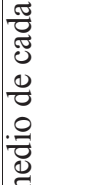 \\
\hline$\frac{n}{ָ}$ & $\begin{array}{l}\vec{N} \\
\stackrel{0}{\dot{1}} \\
\dot{0}\end{array}$ & $\begin{array}{l}\infty \\
\stackrel{\infty}{R} \\
\dot{1}\end{array}$ & $\begin{array}{l}n \\
\tilde{n} \\
\tilde{g} \\
\tilde{o}\end{array}$ & $\begin{array}{l}0 \\
8 \\
0 \\
-i \\
1\end{array}$ & $\begin{array}{c}\overrightarrow{0} \\
\tilde{\sigma} \\
\dot{\alpha} \\
\dot{0}\end{array}$ & $\begin{array}{l}\infty \\
\widetilde{n} \\
0 \\
0 \\
\dot{0}\end{array}$ & 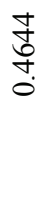 & $\begin{array}{l}\vec{\sigma} \\
\sigma \\
\tilde{\sigma} \\
0\end{array}$ & $\left|\begin{array}{c}\vec{\infty} \\
0 \\
\sim \\
\sim \\
-1\end{array}\right|$ & $\begin{array}{l}n \\
n \\
\hat{\alpha} \\
\dot{p}\end{array}$ & 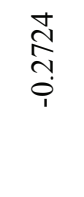 & 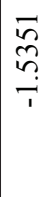 & 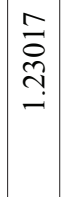 & 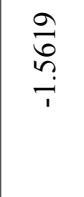 & \begin{tabular}{l}
$\hat{a}$ \\
\multirow{+}{t}{} \\
$\dot{0}$ \\
1
\end{tabular} & $\begin{array}{l}\stackrel{m}{*} \\
\stackrel{+}{n} \\
\stackrel{1}{1}\end{array}$ & 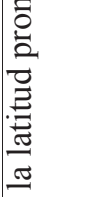 \\
\hline \begin{tabular}{l}
$\infty$ \\
\multirow{0}{0}{} \\
0 \\
$\dot{0}$
\end{tabular} & $\begin{array}{l}2 \\
20 \\
8 \\
0 \\
0\end{array}$ & $\begin{array}{l} \pm \\
\pm \\
\\
\dot{P}\end{array}$ & \begin{tabular}{l}
$n$ \\
\multirow{2}{*}{} \\
0 \\
$i$ \\
$i$
\end{tabular} & 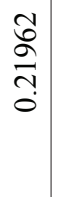 & 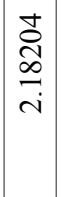 & $\begin{array}{l}0 \\
\text { ర్ర } \\
\stackrel{0}{0} \\
i\end{array}$ & 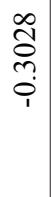 & 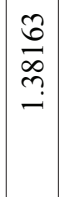 & 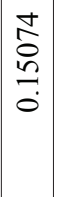 & 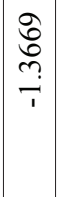 & $\begin{array}{l}\text { ते } \\
\text { ते } \\
\stackrel{1}{1}\end{array}$ & 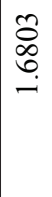 & $\frac{\vec{n}}{\stackrel{0}{0}}$ & $\begin{array}{l}\underset{+}{\infty} \\
\stackrel{2}{\circ} \\
\stackrel{\infty}{+}\end{array}$ & $\begin{array}{l}N \\
\hat{N} \\
\infty \\
\dot{0} \\
1\end{array}$ & 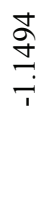 & $\begin{array}{l}0 \\
0 \\
0 \\
0 \\
0 \\
0 \\
0 \\
0 \\
\pi \\
\vdots \\
0\end{array}$ \\
\hline 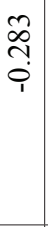 & $\begin{array}{l}0 \\
0 \\
0 \\
n \\
0 \\
1\end{array}$ & $\begin{array}{l}\tilde{T} \\
\stackrel{T}{\infty} \\
\dot{0} \\
1\end{array}$ & $\begin{array}{l}\tilde{N} \\
\tilde{o} \\
- \\
-\end{array}$ & 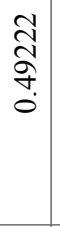 & \begin{tabular}{|l|}
$\infty$ \\
0 \\
$\infty$ \\
$\infty$ \\
+ \\
0 \\
0
\end{tabular} & $\underset{0}{\stackrel{N}{N}}$ & $\begin{array}{l}\hat{n} \\
\stackrel{n}{\vdots} \\
0\end{array}$ & 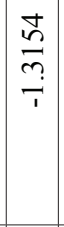 & $\begin{array}{l}0 \\
\infty \\
\infty \\
\infty \\
0 \\
-1 \\
-\end{array}$ & $\begin{array}{l}\stackrel{m}{n} \\
\stackrel{n}{p} \\
\stackrel{1}{1}\end{array}$ & $\begin{array}{l}\stackrel{0}{2} \\
\stackrel{0}{0}\end{array}$ & $\frac{\stackrel{2}{\hat{~}}}{\stackrel{2}{0}}$ & $\begin{array}{l}2 \\
\tilde{N} \\
\tilde{\sigma} \\
\hat{T}\end{array}$ & 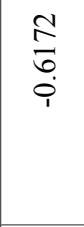 & 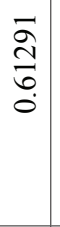 & 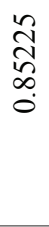 & 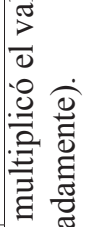 \\
\hline \begin{tabular}{l}
$\infty$ \\
\multirow{2}{n}{} \\
$\infty$ \\
0 \\
0
\end{tabular} & $\begin{array}{c}\stackrel{0}{ } \\
\infty \\
\stackrel{-}{-1} \\
\end{array}$ & $\begin{array}{l}\stackrel{ \pm}{d} \\
\tilde{C} \\
i \\
i\end{array}$ & $\underset{\stackrel{8}{0}}{\stackrel{2}{0}}$ & 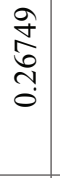 & $\begin{array}{l}\vec{b} \\
0 \\
0 \\
0 \\
1\end{array}$ & $\frac{\hat{a}}{i n}$ & $\begin{array}{l}\overrightarrow{0} \\
\vec{\sigma} \\
\tilde{c} \\
0\end{array}$ & $\begin{array}{l}n \\
\hat{n} \\
\infty \\
0 \\
0\end{array}$ & \begin{tabular}{l}
$n$ \\
2 \\
$y$ \\
\multirow{2}{*}{} \\
0 \\
1
\end{tabular} & 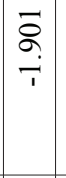 & 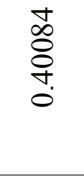 & $\begin{array}{l} \pm \\
\Xi \\
0 \\
0 \\
0\end{array}$ & $\left|\begin{array}{c}n \\
\stackrel{n}{n} \\
\end{array}\right|$ & 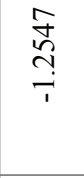 & $\begin{array}{l}0 \\
: \\
n \\
\infty \\
0 \\
1\end{array}$ & $\begin{array}{l}\stackrel{+}{n} \\
\stackrel{n}{n}\end{array}$ & 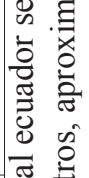 \\
\hline $\begin{array}{l}\frac{D}{\sigma} \\
\vdots \\
0 \\
0\end{array}$ & $\begin{array}{l}m \\
\stackrel{0}{\sigma} \\
\dot{1}\end{array}$ & 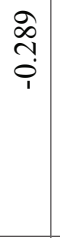 & $\begin{array}{l}\hat{N} \\
\delta \\
0 \\
+ \\
i\end{array}$ & $\begin{array}{c}\vec{\infty} \\
\stackrel{N}{y} \\
\tilde{n} \\
0\end{array}$ & 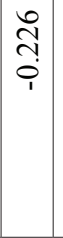 & 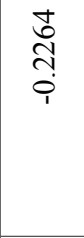 & \begin{tabular}{l}
$n$ \\
$\tilde{n}$ \\
$n$ \\
\hdashline \\
$T$
\end{tabular} & $\left|\begin{array}{l}n \\
2 \\
\delta \\
o \\
i\end{array}\right|$ & 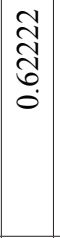 & $\begin{array}{l}\vec{n} \\
\hat{0} \\
0 \\
0\end{array}$ & $\begin{array}{l}\infty \\
\underset{N}{N} \\
\infty \\
\dot{\varphi}\end{array}$ & $\begin{array}{l}\tilde{\infty} \\
\infty \\
\tilde{0} \\
\dot{0}\end{array}$ & 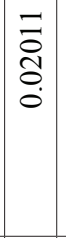 & 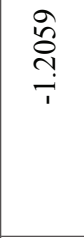 & $\begin{array}{l}\infty \\
\infty \\
\infty \\
\infty \\
? \\
-1\end{array}$ & 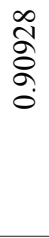 & 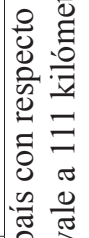 \\
\hline $\begin{array}{l}9 \\
\stackrel{2}{6} \\
\text { bे } \\
\dot{0}\end{array}$ & $\begin{array}{c}0 \\
\infty \\
0 \\
\dot{1}\end{array}$ & $\begin{array}{l}\vec{a} \\
\dot{\sigma} \\
\dot{0} \\
1\end{array}$ & $\begin{array}{l}0 \\
0 \\
n \\
n \\
0 \\
1\end{array}$ & $\begin{array}{c}2 \\
\tilde{n} \\
n \\
0 \\
1\end{array}$ & $\begin{array}{c}n \\
\hat{n} \\
\tilde{o} \\
\dot{p} \\
1\end{array}$ & 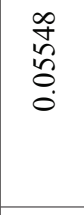 & \begin{tabular}{l}
$\forall$ \\
\multirow{2}{*}{} \\
$\infty$ \\
0 \\
1
\end{tabular} & $\begin{array}{l}\vec{N} \\
\tilde{n} \\
\tilde{i}\end{array}$ & $\mid \begin{array}{l}\hat{\infty} \\
\tilde{\alpha} \\
\hat{\sigma} \\
\dot{0}\end{array}$ & 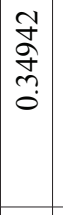 & 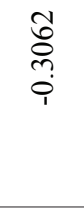 & $\begin{array}{l}\text { 守 } \\
n \\
n \\
0\end{array}$ & 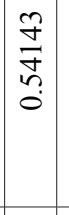 & $\begin{array}{l}\stackrel{n}{\Xi} \\
\equiv \\
0\end{array}$ & 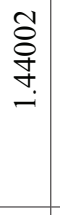 & 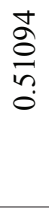 & 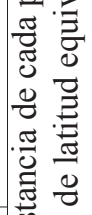 \\
\hline $\begin{array}{l}n \\
\stackrel{n}{2} \\
\hat{0} \\
i\end{array}$ & $\begin{array}{l}\hat{b} \\
\infty \\
n \\
? \\
i\end{array}$ & $\frac{\hat{2}}{8}$ & \begin{tabular}{c} 
I \\
$\exists$ \\
\hdashline \\
\end{tabular} & 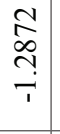 & $\begin{array}{l}\infty \\
\infty \\
\infty \\
0 \\
0\end{array}$ & $\begin{array}{l}\stackrel{a}{0} \\
\frac{1}{1}\end{array}$ & $\begin{array}{l}\stackrel{ \pm}{N} \\
\tilde{n} \\
0 \\
1\end{array}$ & $\left|\begin{array}{c}n \\
\tilde{s} \\
\multirow{2}{t}{} \\
\dot{n}\end{array}\right|$ & $\begin{array}{c}\stackrel{0}{\circ} \\
\stackrel{+}{+} \\
\\
0\end{array} \mid$ & 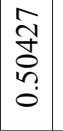 & $\begin{array}{l}\hat{0} \\
\hat{o} \\
\dot{i}\end{array}$ & $\begin{array}{l}m \\
\tilde{n} \\
\tilde{I} \\
0\end{array}$ & $\begin{array}{l}\infty \\
\infty \\
\infty \\
\tilde{0} \\
\tilde{n} \\
0\end{array}$ & $\begin{array}{l}\tilde{n} \\
\text { ஸे } \\
\tilde{\infty} \\
\tilde{0}\end{array}$ & $\begin{array}{l}\vec{\infty} \\
\stackrel{\infty}{-} \\
\Xi\end{array}$ & $\frac{+}{\infty}$ & 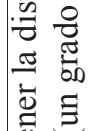 \\
\hline $\begin{array}{l}\cdot \frac{\pi}{\vec{D}} \\
.00 \\
\bar{Z}\end{array}$ & 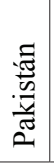 & 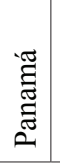 & 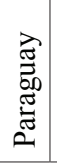 & $\begin{array}{l}\vec{Z} \\
0 \\
2\end{array}$ & 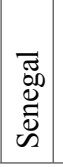 & 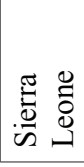 & 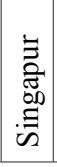 & 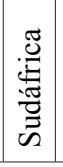 & 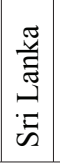 & 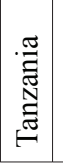 & 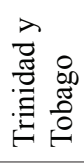 & $\cdot \stackrel{\frac{\pi}{a}}{\Xi}$ & 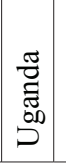 & 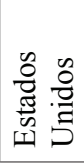 & 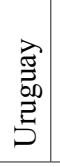 & $\begin{array}{l}\frac{\pi}{0} \\
\frac{0}{0} \\
\frac{0}{0}\end{array}$ & 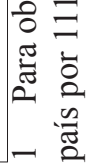 \\
\hline
\end{tabular}

Enero - junio de 2014 - Págs. 11-36 